05348

1996

044

Serie Research Memoranda

Duration Dependence and Heterogeneity in

French Youth Unemployment Durations

Gerard J. van den Berg

Jan C. van Ours

Research Memorandum 1996-44

September 1996

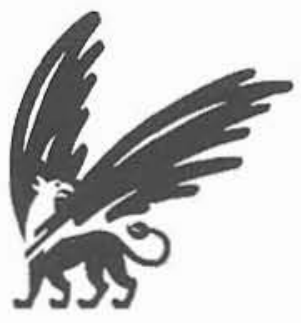




\title{
DURATION DEPENDENCE AND HETEROGENEITY IN FRENCH YOUTH UNEMPLOXMENT DURATIONS
}

\author{
Gerard J. van den Berg* \\ Jan C. van Ours**
}

\begin{abstract}
This paper investigates the degree in which the individual exit rate out of unemployment for young job seekers changes as a function of the elapsed unemployment duration. We use a nonparametric estimation method that is designed to be applicable to population data on outflows from different duration classes. The method also provides estimates of the distribution of unobserved heterogeneity in these data. The model and estimation method explicitly take into account that individual exit rates are affected by the business cycle. The method is applied to population data on young French unemployed job seekers. The results are used for policy recommendations.
\end{abstract}

Date: September 27, 1996.

*Dept. of Economics, Free University Amsterdam, De Boelelaan 1105,

NL-1081 HV Amsterdam, The Netherlands, CEPR, and Tinbergen Institute.

E-mail: gberg@econ.vu.nl

${ }^{* *}$ Erasmus University Rotterdam, CEPR, and Tinbergen Institute.

E-mail: ours@tir.few.eur.nl

Financial support from the Commissariat Général du PLAN in Paris, France is gratefully acknowledged. The Département du Marché du Travail of the Ministère du Travail, de l'Emploi et de la Formation Professionnelle of France kindly provided the data. Thanks to Jaap Abbring for computational assistance. 


\section{Introduction.}

Youth unemployment is among the top policy issues in Western European countries. It is regarded to be relatively undesirable for economic as well as for sociological reasons. Young unemployed job seekers are not yet firmly rooted in the labor market. Unemployment may reduce the human capital they acquired while at school, and it may reduce their commitment to society as a whole. In most OECD countries, including France, the unemployment rates of men and women aged below 25 have increased substantially during the eighties and nineties (OECD, (1996)). This is even more worrying in the light of the fact that, at the same time, the cohort sizes of youths have decreased for demographic reasons, which by itself should have raised the employment prospects of the youth.

Because of all this, it is thought to be important to direct efforts towards a reduction of youth unemployment. Specific policy programs include training programs aimed at reschooling, the creation of apprenticeship positions, youth minimum wages, job search supervision programs, and the enforcement of benefits entitlement requirements by means of punitive sanctions for young benefits recipients. (For examples of empirical analyses of such programs, see Bonnal, Fougère and Sérandon (1994) for training programs and apprenticeships, Van den Berg and Ridder (1993) for youth minimum wages, and Abbring, Van den Berg and Van Ours (1996) for sanctions.)

In this paper we examine the determinants of the unemployment durations of young job seekers. Specifically, we examine to what extent the individual exit probability out of unemployment for young job seekers changes as a function of the elapsed unemployment duration (i.e. to what extent it displays duration dependence). According to economic theory, there are reasons to believe that this probability decreases as a function of duration. For example, there may be stigma effects reducing the number of job opportunities for the long-term unemployed (see e.g. Vishwanath (1989) and Van den Berg (1994)). Such stigma may originate from employers' beliefs concerning the loss of skills (human capital) during unemployment. Also, individuals may become discouraged after a certain period of job search, and their search intensity may decrease as a result of this. If the individual exit probabilities decrease as a function of duration then the aggregate exit probability also decreases as a function of duration (with "aggregate" we mean that we jointly consider individuals with the same observable characteristics but possibly different unobserved characteristics). However, the aggregate exit probability may also decrease for another reason. If certain unobserved individual characteristics 
affect the individual exit probability then on average individuals with the largest individual exit probability leave unemployment first. This causes the aggregate exit probability to decrease as well. In other words, unobserved heterogeneity causes the hazard of the duration distribution of individuals with the same observable characteristics to decrease as well. Since both factors affect the aggregate (or "observed") exit probability in a similar way, it seems to be hard to distinguish empirically between them.

Obviously, from a policy point of view, it is important to know the relative importance of duration dependence on the one hand, and unobserved heterogeneity on the other. If there is negative duration dependence (i.e. if all individual exit probabilities decrease over the duration) then presumably there are stigma or discouragement effects associated to being long term unemployed, and policy should be directed towards preventing workers becoming long term unemployed. If regular job search advice increases the individual exit probability by say $20 \%$ then this is obviously more productive when the baseline exit probability is still relatively high. Knowledge of the duration at which individual exit probabilities start to decrease is important as well. If these exit probabilities do not start to decrease before one year then it is cheaper to offer job search advice only to those that are unemployed for more than say 6 months, in comparison to when this would be offered to all unemployed. This is simply a consequence of the fact that a number of unemployed individuals find a job without help before they reach this 6 month duration.

If unobserved heterogeneity is important, then it may be useful to screen the individuals flowing into unemployment in order to try to observe the corresponding characteristics, and to concentrate efforts on those with bad characteristics. The latter individuals may then be allocated to specific training activities like reschooling.

Generally, studies trying to estimate duration dependence while dealing with unobserved heterogeneity use micro survey data on unemployment durations. From the end of the 1970s onwards a large quantity of reduced form empirical studies have been carried out (for a survey, see Devine and Kiefer (1991)). They are characterized by parametric assumptions on the model specification. Since in general these assumptions are based on analytical tractability rather than strong prior information, such analyses may give biased results on duration dependence.

In this paper, we apply a method for the nonparametric estimation of duration dependence and unobserved heterogeneity that has been developed by Van den Berg and Van Ours (1996). This method is designed to be applicable to 
discrete-time time-series data on gross outflows from different unemployment duration classes. Gross data have the advantage that they provide the exact values of the exit probabilities out of the different duration classes considered (averaged over unobserved heterogeneity). The model and the estimation method explicitly take into account that individual exit rates are affected by macro effects like business-cycle effects and seasonal effects. It is well known that youth unemployment is very sensitive to cyclical fluctuations (see e.g. OECD (1996)).

In the empirical analysis we use French administrative data which distinguish unemployment by elapsed duration and by gender. The data are quarterly, and they cover the period 1982-1992. We use the information on individuals aged below 25 .

The plan of the paper is as follows. Section 2 presents the model and the estimation method. In Section 3 we describe the data. Section 4 presents the results of the analysis. We discuss the parameter estimates and their policy implications. Section 5 concludes.

\section{The model and the estimation method.}

\subsection{The model for the individual exit probability out of unemployment.}

In this section we present the model and the estimation method. Since these are based on Van den Berg and Van Ours (1996), the exposition will be brief.

The present subsection deals with the unemployment duration model and the underlying assumptions. We use two measures of time, each with a different origin. The variable $t$ denotes the duration of a spell of unemployment for a given individual. The variable $\tau$ denotes calendar time. We take $t$ and $\tau$ to have the same measurement scale, apart from the difference in origin. Both $t$ and $\tau$ are discrete variables. For example, consider an individual who is unemployed for $t$ periods at calendar time $\tau$. If he fails to leave unemployment in period $t$, he will be unemployed for $t+1$ periods at calendar time $\tau+1$.

For a good understanding of the model and the estimation method, it is useful to have an idea of the data to which they will be applied.Aggregate data give the total numbers of individuals in the labor market who are unemployed for $t$ periods of time $(t=0,1,2, \ldots)$ at calendar times $\tau$ $\left(\tau=\tau_{0}, \tau_{0}+1, \tau_{0}+2, \ldots\right)$. By comparing the number of individuals who are unemployed for $t$ periods of time at $\tau$ to the number unemployed for $t+1$ periods at $\tau+1$, we observe the fraction of the former who leave unemployment at $\tau$. 
This fraction of course equals the probability that a randomly chosen individual who is unemployed for $t$ periods leaves unemployment, when calendar time equals $\tau$ at the moment of potential exit. So, we observe these exit probabilities for different values of $t$ and $\tau$.

The model for these exit probabilities expresses them in terms of the (determinants of the) exit probabilities at the individual level. The relation is established by way of aggregating over individual unemployment duration distributions. It is assumed that all variation in the individual exit probabilities out of unemployment can be explained by the prevailing unemployment duration $t$ and calendar time $\tau$, and by observed and unobserved heterogeneity across individuals. The effect of $t$ represents genuine duration dependence, i.e. dependence of individual exit probabilities on the elapsed unemployment duration. Calendar time is assumed to capture macro effects (including business cycle and seasonal effects) on individual exit probabilities, as well as structural changes influencing these probabilities.

In the data we will use, gender is the only observed individual characteristic that can be used as an explanatory variable $x$. We therefore estimate the model separately for both genders, and in the sequel we suppress the conditioning on the prevailing value of $x$.

We denote the probability that an individual leaves unemployment right after $t$ periods of unemployment, given that he is unemployed for $t$ periods at calendar time $\tau$, and conditional on his unobserved characteristics $v$, by $\theta(t \mid \tau, v)$. By definition, this is the exit probability out of unemployment (or hazard) at $t$ conditional on $\tau$ and $v$. The unemployment duration density conditional on calendar time and conditional on $v$ can be constructed from these individual exit probabilities. For example, the probability that unemployment duration equals $t$, when calendar time was $\tau-t$ at the moment of inflow into unemployment, conditional on $v$, equals

$$
\theta(t \mid \tau, v) \cdot \prod_{i=1}^{t}(1-\theta(t-i \mid \tau-i, v))
$$

for all $t \in\{0,1, .$.$\} . We take the product term to be one if t=0$.

We make the following assumptions.

\section{Assumptions}

1. MPH: $\theta(t \mid \tau, v)$ has a mixed proportional hazard specification, i.e. there are functions $\psi_{1}$ and $\psi_{2}$ such that

$$
\theta(t \mid \tau, v)=\psi_{1}(t) \cdot \psi_{2}(\tau) . v
$$


with $\psi_{1}$ and $\psi_{2}$ positive and uniformly bounded from above. Further, the distribution of $v$ is such that, for every $t$ and $\tau$, $\operatorname{Pr}(0<\theta(t \mid \tau, v)<1)=1$.

2. Independence of $v$ and $\tau$ : the distribution $G(v)$ in the inflow into unemployment does not depend on the moment of inflow (this part will be relaxed later on). Further, the individual level of $v$ does not change during unemployment.

3. Variation over calendar time: the function $\psi_{2}$ is not constant.

The functions $\psi_{1}$ and $\psi_{2}$ represent the duration dependence and the calendar time dependence of the individual exit probabilities out of unemployment. As shown by Van den Berg and Van Ours (1996), Assumptions 1-3 ensure nonparametric identifiability of the model. In particular, they ensure that duration dependence and unobserved heterogeneity can be distinguished empirically without the need to specify parametric functional forms on the shape of $\psi_{1}$ or $G$.

Assumption 1 is reminiscent of the standard MPH assumption in reduced-form models for micro duration data (see Lancaster (1990) for an extensive survey of such models). In models for micro duration data, dependence on calendar time is usually ignored, and the role of $\tau$ in the model above is replaced by the role of observed explanatory variables $x$. Elbers \& Ridder (1982) prove that the latter type of models are nonparametrically identified if assumptions similar to above are satisfied. Whenever calendar time is included as a regressor in reduced-form duration models for micro data, it is usually included as a multiplicative term in the hazard rate (see e.g. Imbens (1994)).

An important difference between the present model and MPH models for micro data is that here we have discrete time, whereas in micro studies time is usually treated as continuous. Note that the present model is not the discretized (i.e. grouped) version of the continuous-time MPH model (see e.g. Meyer (1990) and McCall (1994)). Indeed, the present model should not be interpreted as an approximation to the continuous-time MPH model. Rather, it should be regarded as a flexible accounting device for discrete aggregate duration data, with an appealing interpretation. Still, as shown in Van den Berg and Van Ours (1996), certain important qualitative features of the present model are the same as those of the continuous-time MPH model.

Because of the discrete-time framework, we had to introduce the last line of Assumption 1. Note that it implies that the support of $v$ is bounded. This in turn implies that all moments of $v$ exist. 


\subsection{Empirical implementation.}

In this subsection we present the strategy to estimate the parameters of interest. As mentioned above, the data provide observations on the probabilities that individuals leave unemployment when being unemployed for $t$ periods, when calendar time equals $\tau$ at the moment of exit, for different values of $t$ and $\tau$. These probabilities are unconditional on the unobserved heterogeneity term $v$, and will be denoted by $\theta(t \mid \tau)$. To express them in terms of the exit probabilities $\theta(t \mid \tau, v)$, we have to integrate $v$ out of the latter. The situation is similar to reduced-form analyses of micro duration data in which the model expresses the exit probabilities conditional on observed and unobserved explanatory variables but the data only provide information on exit probabilities conditional on the observed variables.

Let $t$ denote the random unemployment duration, and $t$ its realization. In obvious notation, there holds that

$$
\theta(t \mid \tau) \equiv \frac{\operatorname{Pr}(t=t \mid \text { inflow at } \tau-t)}{\operatorname{Pr}(t \geq t \mid \text { inflow at } \tau-t)} \equiv \frac{E_{v}(\operatorname{Pr}(t=t \mid \text { inflow at } \tau-t ; v))}{\mathrm{E}_{v}(\operatorname{Pr}(t \geq t \mid \text { inflow at } \tau-t ; v))}
$$

in which $E_{v}($.$) denotes the expectation with respect to the distribution G$ of $v$ in the inflow into unemployment, and in which $\operatorname{Pr}(t=t \mid$ inflow at $\tau-t ; v)$ and $\operatorname{Pr}(t \geq t \mid$ inflow at $\tau-t ; v)$ can be expressed in terms of $\theta(t \mid \tau, v)$ (note that equation (2.1) gives $\operatorname{Pr}(t=t \mid$ inflow at $\tau-t ; v))$. By doing this, and by substituting equation (2.2), we get

$$
\theta(t \mid \tau)=\frac{\psi_{1}(t) \cdot \psi_{2}(\tau) \cdot \mathrm{E}_{v}\left[v \cdot \prod_{i=1}^{t}\left[1-\psi_{1}(t-i) \cdot \psi_{2}(\tau-i) \cdot v\right]\right]}{\mathrm{E}_{v}\left[\prod_{i=1}^{t}\left[1-\psi_{1}(t-i) \cdot \psi_{2}(\tau-i) \cdot v\right]\right]}
$$

Thus, $\theta(t \mid \tau)$ can be expressed in terms of the "structural functions" $\psi_{1}, \psi_{2}$ and the distribution function $G(v)$ of $v$. In fact, we can be more specific on the way $G(v)$ enters such expressions. By expanding the products in the r.h.s. of (2.4) it follows that $\theta(t \mid \tau)$ depends on $G(v)$ only by way of the first $t+1$ moments of $v$. Denote $\mathrm{E}_{v}\left(v^{i}\right)$ by $\mu_{i}$. We have the following result: $\theta(t \mid \tau)$ depends on $\left\{\psi_{1}(i), \psi_{2}(\tau-t+i), \mu_{i+1}\right.$, with $\left.i=0,1, . ., t\right\}$. We will call the elements of the latter set the "parameters", even though they really are values of functions on $\mathbb{N}$ and summary statistics of the underlying heterogeneity distribution, respectively. 
Typical samples contain information on $\theta(t \mid \tau)$ for a small number $n_{t}$ of different durations and a large number $n_{\tau}$ of different points of time. In such cases, the number of parameters is large relative to the number of observations. In particular, for each $\psi_{2}(\tau)$ parameter the number of observations that contain information on that parameter is extremely small, so the estimate of it would be unreliable. However, note that we are primarily interested in estimating the duration dependence and unobserved heterogeneity parameters and that the calendar time dependence parameters are nuisance parameters. Van den Berg and Van Ours (1996) present a strategy for estimating the parameters of interest only. Basically, these ideas amount to substituting values of past observed exit probabilities into the expressions (2.4) for $\theta(t \mid \tau)$, and examining ratios of the resulting expressions for different $t$.

As an example, consider $\theta(1 \mid \tau)$. From (2.4), it follows that this is equal to $\psi_{1}(1) \cdot \psi_{2}(\tau) \cdot\left(\mu_{1}-\psi_{1}(0) \cdot \psi_{2}(\tau-1) \cdot \mu_{2}\right) /\left(1-\psi_{1}(0) \cdot \psi_{2}(\tau-1) \cdot \mu_{1}\right)$. But from $(2.4)$ it also follows that $\psi_{1}(0) \cdot \psi_{2}(\tau-1)$ equals $\theta(0 \mid \tau-1) / \mu_{1}$. Consequently,

$$
\frac{\theta(1 \mid \tau)}{\theta(0 \mid \tau)}=\frac{\psi_{1}(1)}{\psi_{1}(0)} \cdot \frac{1-\theta(0 \mid \tau-1) \cdot \mu_{2} / \mu_{1}^{2}}{1-\theta(0 \mid \tau-1)}
$$

in which $\psi_{2}$ does not show up anymore.

Denote $\mu_{i} / \mu_{1}^{i}$ by $\gamma_{i}(i \geq 2)$, and $\psi_{1}(t) / \psi_{1}(t-1)$ by $\eta_{t}(t \geq 1)$. The parameters $\eta_{t}$ represent the duration dependence of the exit probability as a function of $t$, whereas the parameters $\gamma_{i}$ represent the normalized moments of the distribution of unobserved heterogeneity. The general result is

$$
\begin{gathered}
\frac{\theta(t \mid \tau)}{\theta(t-1 \mid \tau)}=\eta_{t} \cdot\left\{\text { expression depending on } \theta(i-1 \mid \tau-t+i-1) \text { and } \gamma_{i+1}\right. \\
\text { with } i=1,2, . ., t, \text { and, if } t \geq 2 \text {, on } \theta(i-1 \mid \tau-t+i) \text { with } i=1,2, . ., t-1\}
\end{gathered}
$$

As a second example, for $t=2$ we get

$$
\begin{aligned}
& \frac{\theta(2 \mid \tau)}{\theta(1 \mid \tau)}=\eta_{2} \cdot \frac{1-\theta(0 \mid \tau-1)}{(1-\theta(1 \mid \tau-1)) \cdot(1-\theta(0 \mid \tau-2))} \\
& \frac{1-\gamma_{2} \cdot \theta(0 \mid \tau-2)-\theta(1 \mid \tau-1) \cdot(1-\theta(0 \mid \tau-2)) \cdot \frac{\gamma_{2}-\gamma_{3} \cdot \theta(0 \mid \tau-2)}{1-\gamma_{2} \cdot \theta(0 \mid \tau-2)}}{1-\gamma_{2} \cdot \theta(0 \mid \tau-1)}
\end{aligned}
$$

Such ratios of observed exit probabilities can be used to estimate the parameters of interest. Note that the nuisance parameters of $\psi_{2}$ do not show up in (2.6), and that the number of parameters does not increase when the number of calendar time points in the data (i.e. the sample size) increases. For each 
parameter $\eta_{t}$ and $\gamma_{i}$, the number of observations that contain information on it is relatively large. If we observe exit probabilities for durations $\left\{0,1, . ., n_{t}-1\right\}$ and calendar times $\left\{T+1, . ., T+n_{\tau}\right\}$ then the number of parameters in the expressions for $\theta(t \mid \tau) / \theta(t-1 \mid \tau)$ equals $2\left(n_{t}-1\right)$.

The parameters are estimated as follows. We specify $\log \theta(t \mid \tau) / \theta(t-1 \mid \tau)$ to equal the log of the corresponding model expression, plus an error term. The error terms represent specification errors that are assumed to be identically distributed over equations and over observations. On the other hand, we allow the errors in different equations to be contemporaneously related. So, at a given point of calendar time, the specification errors for different ratios of exit probabilities may be related. We do not make a parametric assumption on the distribution of the error terms. The estimation method we employ is Seemingly Unrelated Nonlinear Regression (SUNR).

Let us return to the ratio $\theta(t \mid \tau) / \theta(t-1 \mid \tau)$. If there is no unobserved heterogeneity, then $\theta(t \mid \tau) / \theta(t-1 \mid \tau)=\eta_{t}$, so these ratios do not depend on $\tau$. (This can be checked by noting that in that case $\mu_{i}=\mu_{1}^{i}$ for every $i \geq 1$, so $\gamma_{i}=1$.) If there is unobserved heterogeneity, then in general these ratios do depend on $\tau$. For example, there holds that $\theta(1 \mid \tau) / \theta(0 \mid \tau)$ depends on $\theta(0 \mid \tau-1)$ if and only if $\gamma_{2} \neq 1$, which in turn holds if and only if there is unobserved heterogeneity. The exit probability $\theta(0 \mid \tau-1)$ varies with $\tau$ by virtue of Assumption 3, so, in sum, $\theta(1 \mid \tau) / \theta(0 \mid \tau)$ varies with $\tau$ if and only if there is unobserved heterogeneity. The ratio $\theta(t \mid \tau) / \theta(t-1 \mid \tau)$ can be thought of as the discrete-time equivalent of the derivative of $\log \theta(t \mid \tau)$ w.r.t. $t$. This means that the parameters associated with the distribution of unobserved heterogeneity are identified from the interaction terms (or cross effects) of $t$ and $\tau$ in $\log \theta(t \mid \tau)$.

In general, the interaction between $t$ and $\tau$ in the observed exit probabilities that is caused by unobserved heterogeneity is such that the observed degree of duration dependence is less negative in a recession than at the top of the business cycle. For example, equation (2.5) implies that, if $\gamma_{2}>1$ and $\eta_{1}<1$, the decrease of $\theta(t \mid \tau)$ when going from $t=0$ to $t=1$ is smaller in a recession $(\theta(0 \mid \tau-1)$ small $)$ than at the top of the cycle $(\theta(0 \mid \tau-1)$ large $)$. This is because in a recession the weeding out of individuals with a high quality (i.e. a large $v$ ) cannot occur as fast as in the other case. Note that the model implication on the interaction sign is testable. In particular, if $\gamma_{2}<1$ then the interaction sign in $(2.5)$ is opposite to above.

Clearly, the MPH assumption is crucial. Van den Berg and Van Ours (1996) provide a number of specification tests. Consider for example the estimates of $\gamma_{2}, . ., \gamma_{n_{t}}$. If the model is correct, then $\gamma_{2}, . ., \gamma_{n_{t}}$ are mutually consistent as 
normalized moments of a distribution with positive bounded support (from zero until the upper bound depending on the functions $\psi_{1}$ and $\psi_{2}$ ). This can be tested for. For example, if $\gamma_{2}<1$ or $\gamma_{3}<\gamma_{2}^{2}$ then there is no distribution with positive support that is able to generate such moments (see Shohat and Tamarkin (1970); for example $\gamma_{2}<1$ would imply $\left.\operatorname{Var}(v)<0\right)$. If these necessary conditions are not violated, then one can usually find a discrete distribution with a finite number of (positive) points of support that is able to generate the $\gamma_{i}$ estimates (see Shohat and Tamarkin (1970) and Lindsay (1989)). It should be noted that in general there will also be non-discrete distributions that are able to generate a given finite set of moments. Consequently, if the estimated $\gamma_{i}$ are normalized moments of some distribution, then in general there will be more than one distribution function $G(v)$ consistent with them. It can be shown that the moment tests are informative on the validity of Assumption 1. Also, they may detect misspecification of the unit of time period.

Before finishing this section we discuss the incorporation of seasonal effects into the model. One may distinguish two types of seasonal effects on the exit probabilities. First, there may be an effect that equally affects every unemployed individual. For example, there may be less activity on the labor market during the holiday season. This effect is captured by the $\psi_{2}(\tau)$ terms in the model. Secondly, there may be a permanent effect of the season prevailing at the moment of inflow into unemployment. For example, the success of individuals in the inflow at the end of the schooling season may on average be different from that at other times of the year. This may be labeled the cohort effect. To incorporate it, we allow for dependence of $G(v)$ on the moment of inflow, so we model the composition of the inflow to be dependent on the season at the moment of inflow. We assume that the season at the moment of inflow affects a scale parameter of the distribution of unobserved heterogeneity in the inflow. For example, for two seasons $A$ and $B$, we assume that $G_{B}(v)=G_{A}(\omega \cdot v)$. If $\omega>1$ then $A$ is a "better" season than $B$. There holds that $\mu_{i, A}=\omega^{i} \cdot \mu_{i, B}$ and $\mu_{i, A} / \mu_{1, A}^{i}=\mu_{i, B} / \mu_{1, B}^{i}$. The latter ratios are again denoted by $\gamma_{i}$.

It can be shown that the only thing that changes in $(2.6)$ is that the r.h.s. has to be multiplied by the scale parameter giving the change in $G(v)$ when going from the season at $\tau-t$ to the season at $\tau-t+1$. For example, if $\tau-t$ is a season of type $A$ and $\tau-t+1$ is a season of type $B$ and $G_{B}(v)=G_{A}(\omega \cdot v)$, then $\theta(t \mid \tau) / \theta(t-1 \mid \tau)$ is equal to a factor $\omega$ times the expression in the r.h.s. of (2.6). Thus, the seasonal effects are estimated along with the other parameters. 
In the application, the duration of a season is taken to equal the unit of time, which is one quarter. Thus, we have four seasons and four additional parameters $\omega_{1}, \omega_{2}, \omega_{3}$ and $\omega_{4}$ defined by $G_{1}(v)=G_{4}\left(\omega_{1} \cdot v\right)$ and $G_{i}(v)=$ $G_{i-1}\left(\omega_{i} . v\right)$ for $i \in\{2,3,4\}$, with $\omega_{4}=1 /\left(\omega_{1} \omega_{2} \omega_{3}\right)$. So, if $\omega_{i}<1$ then the average success of the newly unemployed workers in season $i$ is larger than in the inflow one quarter earlier.

\section{Data.}

The data we use are French administrative data on individuals aged below 25, for both genders. These were collected by the French public employment offices (A.N.P.E.), and subsequently collected on a nation-wide scale by the Department of Labor. They cover individuals who are looking for full-time permanent jobs.

For the model to be applicable, the frequency at which the data are collected has to equal one over the size of the unemployment duration classes (i.e. the unit time period in the model). We use quarterly data on the exit probabilities out of the first four quarterly duration classes, over the period 1982.1-1992.1.

The way in which the observed exit probability out of unemployment $\theta(t \mid \tau)$ behaves as a function of $t$ differs across gender, in some respects. First of all, the observed exit probability is generally smaller for females than for males, at all durations. Secondly, the observed exit probability is often more decreasing for females than than for males. Whether this is due to differences in duration dependence or to differences in the degree of unobserved heterogeneity cannot be answered at this stage. In any case, the two differences above imply that in general the gap between the exit probabilities for males and females is widening as unemployment duration proceeds. A somewhat more detailed description of the data is in Van den Berg and Van Ours (1994).

The information about the first four exit probabilities allows us to estimate three equations like (2.6) (each $t \in\{1,2,3\}$ defines one equation). These contain 3 heterogeneity parameters $\left(\gamma_{2}, \gamma_{3}, \gamma_{4}\right), 3$ duration dependence parameters $\left(\eta_{1}, \eta_{2}, \eta_{3}\right)$, and 3 seasonal-effect parameters $\omega_{1}, \omega_{2}$ and $\omega_{3}$ (note that $\omega_{4}$ follows from these). 


\section{Estimation results.}

The estimation results are shown in Table 1 . We start by discussing the $\eta_{t}$ estimates. These indicate that for young women there is negative duration dependence of the exit probability out of unemployment, in particular after the second quarter of unemployment. For young women, the exit probability after one year of unemployment is $87 \%$ of the exit probability in the first quarter of unemployment. This may be due to a stigma effect of not being short-term unemployed anymore, or due to a discouragement effect of not having been able to find a job yet.

For young male workers there is no significant duration dependence during the first year of unemployment. This result, and the fact that it differs from the result on duration dependence for young women, can be explained in a number of ways. First of all, if the schooling level is on average higher for men, then a certain loss of human capital during unemployment leaves long-term unemployed men with still a higher average level of human capital than long-time unemployed women, so that those men can still pursue a wider range of job opportunities, and they have less of a stigma. Another explanation could be that men on average have a lower non-pecuniary utility of being long-term unemployed than women, for example because men on average may find it harder to create non-work activities. If such activities replace time devoted to job search, then the latter can be rephrased by stating that long-time unemployed women may more often make a transition to nonparticipation that is not reported as such (i.e. that they make such a transition while still registering themselves as being unemployed). In the absence of additional information it is hard to assess the power of such possible explanations. (Note that in the micro-econometric literature on unemployment durations it is always assumed that the duration dependence parameters do not depend on individual characteristics like gender.)

The $\gamma_{i}$ estimates show that there is significant unobserved heterogeneity, for each gender. All $\gamma_{i}$ estimates are significantly larger than one. Further, $\gamma_{3}-\gamma_{2}^{2}$ is significantly larger than zero whereas $\left(\gamma_{4}-\gamma_{2}^{2}\right) \cdot\left(\gamma_{2}-1\right)-\left(\gamma_{3}-\gamma_{2}\right)^{2}$ does not differ significantly from zero, for each gender. Using results in Shohat \& Tamarkin (1970), this implies that, for each gender, $G(v)$ can be accurately described by a discrete distribution with two positive points of support. The estimates of $\gamma_{2}$ do not differ significantly across gender, so the coefficient of variation of unobserved heterogeneity for males is approximately the same as for females.

It may be interesting to infer the specification of the implied discrete 
unobserved heterogeneity distribution. For males it turns out that $76 \%$ of the inflow into unemployment has a heterogeneity value equal to 0.82 times the mean $\mu_{1}$ of the heterogeneity distribution, and $24 \%$ has a heterogeneity value equal to 1.55 times this mean (so $\operatorname{Pr}\left(v=0.82 \mu_{1}\right)=0.76$ and $\operatorname{Pr}\left(v=1.55 \mu_{1}\right)=0.24$; it is easily confirmed that $\mu_{1}$ is indeed the mean of this distribution). For females, $\operatorname{Pr}\left(v=0.91 \mu_{1}\right)=0.95$ and $\operatorname{Pr}\left(v=2.51 \mu_{1}\right)=0.05$. Thus, for females there is a very small subgroup with a very large exit probability, but outside of this successful subgroup there is no heterogeneity with respect to the exit probability. Since it is unlikely that the level of the exit probability of the vast majority of women can be lifted to the level of this subgroup by way of a policy change, this means that unobserved heterogeneity among women is not very relevant for policy purposes. For men, the size difference between both subgroups is not as large as for women. In both cases, over the duration of unemployment, the size of the group with the higher $v$ (i.e. with the higher exit probability) declines more rapidly than the size of the other group. After about 7 quarters for men, and after about 2 quarters for women, only the group with the smallest exit probability remains.

Our empirical analysis is based on the exit probabilities out of the first four quarters of unemployment. We will now present a somewhat informal procedure to extend this analysis to higher duration classes. As argued above, unobserved heterogeneity can be characterized by a distribution with two points of support. Assuming that these estimated distributions with two points of support are the true heterogeneity distributions, we can extend our empirical analysis to higher duration classes without much computational burden. Along this line, we estimate the $\eta_{t}$ parameters, for $t=4,5, . ., 9$, using data on exit probabilities for 10 (quarterly) duration classes. The parameter estimates are in Table 2. It should be noted that the reported standard errors are conditional on the estimates of the discrete heterogeneity distribution and the seasonal effects parameters. It turned out to be computationally infeasible to estimate the whole model simultaneously.

It turns out that for men there is substantial negative duration dependence after 5 quarters. For women, the negative duration dependence in the third and fourth quarter is followed by a more or less equally strong negative duration dependence at higher durations.

The moment-inequality specification tests that we discussed in Subsection 2.2 indicate that the model specification cannot be rejected, for each gender. The fact that the $\gamma_{2}$ estimates significantly exceed one means that the data confirm that observed duration dependence (when going from $t=0$ to $t=1$ ) is more negative in the top of the cycle than in a recession. Blanchard \& Diamond 
(1994)'s so-called ranking model of unemployment predicts the opposite result. Apparently, in our data, the dynamic selection due to unobserved heterogeneity is empirically more important than ranking phenomena.

From Table 1 it also follows that there are seasonal effects in the heterogeneity distribution. Young individuals who become unemployed in the second part of the year have more success in leaving unemployment than those who become unemployed in the first part of the year.

We finish the discussion of our results by comparing them to the results in Van den Berg and Van Ours (1994). In that study, an analysis similar to the analysis of the present paper was carried out for the whole populations of male and female unemployed in France, under the assumption that the model setup of Section 2 is valid for these two populations. Note that the latter implies that, in that study, $\eta_{t}$ and $\gamma_{i}$ are assumed to be identical across age groups. If the latter is not true then the model in Van den Berg and Van Ours (1994) is misspecified, so any comparison of results should be viewed with caution. With this in mind, we conclude that the main differences are as follows. First of all, and not surprisingly, there appears to be more unobserved heterogeneity in the whole population of a given gender than in the sub-population of young individuals of that gender. Secondly, concerning duration dependence for men, the exit probability for young men does not drop as much when going from the second to the third quarter than the exit probability for the whole population. As a result of this, for young men duration dependence is not as much of a problem as it is for the whole population of unemployed men. Thirdly, duration dependence for females seems to be absent in the fourth quarter when considering the whole population, while this is definitely not true when restricting attention to young women.

In the next section we discuss the policy implications of the estimation results.

\section{Conclusion and policy recommendations.}

In this paper we analyze unemployment dynamics among young job seekers in the French labor market. In particular, we estimate the duration dependence of the exit probability out of unemployment while controlling for unobserved heterogeneity, without imposing parametric functional forms on either of these. The empirical analysis is carried out separately for young men and young women, using aggregate time-series data on outflows from different unemployment duration classes. 
For young women we find negative duration dependence, i.e. a decline of the individual exit probability over the duration of unemployment, in particular after the second quarter of unemployment. For young men there is no significant duration dependence during the first year of unemployment, but we find some evidence of negative duration dependence thereafter. From this we conclude that, for young women, stigma or discouragement effects become relevant relatively quickly after the moment of inflow into unemployment. For young men, these effects become relevant only after having been unemployed for at least one year.

Now consider the implications for policies that are directed towards preventing individuals become long term unemployed, like regular job search advice, job application training, and other training programs. For young women, such policies should focus on individuals who still have a relatively short unemployment duration (between 3 and 6 months). These policies should aim at targeting all individuals in this duration class. For young men, policies that are supposed to prevent long term unemployment do not have to focus on individuals with such short unemployment durations. Instead, such policies could focus on all individuals with an elapsed unemployment duration of say 9 to 12 months. It should be noted that if negative duration dependence for young women merely reflects a voluntary transition from unemployment to nonparticipation that is not registered in the data, then of course a policy against negative duration dependence for this group is not useful.

For young men as well as for young women we find significant unobserved heterogeneity in the unemployment duration distribution. This is hardly surprising, given the degree of aggregation in the data. However, the estimation results imply that it is not useful to pay attention in policy to unobserved heterogeneity among young women. There is a very small subgroup of young women with a very large exit probability, but outside of this successful subgroup there is no heterogeneity among young women with respect to the exit probability. It is unlikely that the level of the exit probability of the vast majority of young women can be lifted to the level of this subgroup by way of a policy change. Among young men, a subgroup consisting of $76 \%$ of the inflow has a substantially lower exit probability than the other subgroup. This means that it may be useful to screen the inflow of young men in order to detect whether individuals belong to the $76 \%$ subgroup, and subsequently concentrate efforts on those individuals.

Note that what is labeled as unobserved heterogeneity in aggregate data may well be partly or fully observable by the employment agency. Micro studies suggest that the level of education, the type of occupation etc. of the 
individual are significant determinants of the exit probability out of unemployment. These variables are observable by the agency, and can therefore be used to screen the male individuals and subsequently allocate those with unfavorable characteristics to certain training programs. 
Table 1

Parameter estimates

(Standard errors between parentheses)

males females

unobserved heterogeneity distribution

\begin{tabular}{|c|c|c|}
\hline$\gamma_{2}$ & $1.099(0.024)$ & $1.129(0.042)$ \\
\hline$\gamma_{3}$ & $1.334(0.079)$ & $1.571(0.140)$ \\
\hline & $1.748(0.150)$ & $2.666(0.309)$ \\
\hline
\end{tabular}

duration dependence

$\begin{array}{llll}\eta_{1} & 1.017(0.029) & 1.023(0.036) \\ \eta_{2} & 0.968(0.020) & 0.913(0.024) \\ \eta_{3} & 0.978(0.025) & 0.938(0.024)\end{array}$

seasonal effect on composition inflow

$\begin{array}{llll}\omega_{1} & 1.056(0.007) & 1.036(0.009) \\ \omega_{2} & 1.003(0.006) & 1.005(0.007) \\ \omega_{3} & 0.966(0.006) & 0.996(0.007) \\ \omega_{4} & 0.977(0.006) & 0.964(0.008)\end{array}$


Table 2

Duration dependence estimates for higher durations, given a discrete heterogeneity distribution with two points of support

(Conditional standard errors between parentheses)

males

$\eta_{4}$

$\eta_{5}$

$\eta_{6}$

$\eta_{7}$

$\eta_{8}$

$\eta_{9}$
$1.021(0.003)$

$0.935(0.006)$

$0.924(0.010)$

$0.936(0.009)$

$0.959(0.014)$

$0.921(0.019)$ females

$0.984(0.005)$

$0.938(0.003)$

$1.001(0.008)$

$0.946(0.008)$

$0.926(0.010)$

$0.923(0.010)$ 


\section{References}

Abbring, J.H., G.J. van den Berg and J.C. van Ours (1996), The effect of unemployment insurance sanctions on the transition rate from unemployment to employment, Working paper, Free University Amsterdam and Tinbergen Institute, Amsterdam.

Blanchard, O.J. and P. Diamond (1994), Ranking, unemployment duration, and wages, Review of Economic Studies 61, 417-434.

Bonnal, L., D. Fougère and A. Sérandon (1994), Estimating the impact of French employment policies on individual labour market histories, Working paper, CREST-INSEE, Paris.

Devine, T.J. and N.M. Kiefer (1991), Empirical Labor Economics, Oxford University Press, New York.

Elbers, C. and G. Ridder (1982), True and spurious duration dependence: the identifiability of the proportional hazard model, Review of Economic Studies 49, 403-410.

Imbens, G.W. (1994), Transition models in a non-stationary environment, Review of Economics and Statistics 76, 703-720.

Lancaster, T. (1990), The econometric analysis of transition data, Cambridge University Press, Cambridge.

Lindsay, B.G. (1989), Moment matrices: applications in mixtures, Annals of Statistics 17, 722-740.

McCall, B. (1994), Specification diagnostics for duration models: a martingale approach, Journal of Econometrics 60, 293-312.

Meyer, B.D. (1990), Unemployment insurance and unemployment spells, Econometrica 58, 757-782.

OECD (1996), Employment outlook 1996, OECD, Paris.

Shohat, J.A. and J.D. Tamarkin (1970), The problem of moments, American Mathematical Society, Providence.

Van den Berg, G.J. (1994), The effects of changes of the job offer arrival rate on the duration of unemployment, Journal of Labor Economics 12, $478-498$.

Van den Berg, G.J. and G. Ridder (1993), An empirical equilibrium search model, Working paper, Free University Amsterdam and Tinbergen Institute, Amsterdam.

Van den Berg, G.J. and J.C. van Ours (1994), Unemployment dynamics and duration dependence in France, The Netherlands and the United Kingdom, Economic Journal 104, 432-443. 
Van den Berg, G.J. and J.C. van Ours (1996), Unemployment dynamics and duration dependence, Journal of Labor Economics 14, 100-125.

Vishwanath, T. (1989), Job search, stigma effect, and escape rate from unemployment, Journal of Labor Economics 7, 487-502. 